\title{
Identification of a Cholinergic Modulatory and Rhythmogenic Mechanism within the Lamprey Respiratory Network
}

\author{
Donatella Mutolo, Elenia Cinelli, Fulvia Bongianni, and Tito Pantaleo \\ Dipartimento di Scienze Fisiologiche, Università degli Studi di Firenze, 50134 Florence, Italy
}

Acetylcholine (ACh) is well known to be involved in the control of breathing. However, no information is available on the role of ACh receptors (AChRs) within the lamprey respiratory network. The present study was performed on in vitro brainstem preparations of adult lampreys to investigate whether ACh affects respiratory activity possibly through an action on the paratrigeminal respiratory group (pTRG) that has been identified as an essential component of the respiratory network. Respiratory activity was monitored as vagal motor output. Bath application of $100 \mu \mathrm{m}$ physostigmine or $1 \mu \mathrm{m}$ nicotine increased respiratory frequency, while bath application of $100 \mu \mathrm{M}$ D-tubocurarine or $0.25 \mu \mathrm{M} \alpha$-bungarotoxin reduced respiratory frequency and increased the duration of vagal bursts. Since these effects were mimicked by microinjections of the same drugs into the pTRG, ACh proved to influence respiratory activity by acting on $\alpha 7$ nicotinic AChRs located within the pTRG. During apnea caused by partial blockade of ionotropic glutamate receptors at the level of the pTRG, bath application of bicuculline and strychnine restored the respiratory rhythm, although at reduced frequency. Similar results were obtained by the concurrent removal of both fast synaptic excitatory and inhibitory transmission. Blockade of pTRG $\alpha 7$ nicotinic AChRs suppressed this respiratory activity, thus indicating that PTRG neurons expressing these receptors contribute to respiratory rhythm generation. Together, these findings identify a novel cholinergic modulatory and possibly subsidiary rhythmogenic mechanism within the respiratory network of the adult lamprey and encourage further studies on the respiratory role of cholinergic receptors in different animal species.

\section{Introduction}

Acetylcholine (ACh) has long been known to play an important role in the neural control of breathing. The most consistent central effect of ACh on respiratory activity has been reported to be excitatory (Bianchi et al., 1995; Shao and Feldman, 2009). Recently, experiments performed on in vitro medullary slices from neonatal rodents (Shao and Feldman, 2000, 2001, 2009) showed that increases in respiratory frequency are produced by the activation of both nicotinic $A C h$ receptors ( $\mathrm{AChRs}$ ) and muscarinic ACh receptors (mAChRs) in the pre-Bötzinger complex (preBötC), a medullary region that has been proposed to play a crucial role in respiratory rhythm generation in mammals (Smith et al., 1991; Feldman and Del Negro, 2006). In particular, the predominant subtype of nAChRs involved in this respiratory effect is an $\alpha 4 \beta 2$ subunit combination (Shao and Feldman, 2002; Shao et al., 2008).

No information is available on the role played by ACh in the control of breathing in the lamprey, a lower vertebrate that di-

\footnotetext{
Received June 3, 2011; revised July 19, 2011; accepted July 26, 2011.

Author contributions: D.M., E.C., F.B., and T.P. designed research; D.M., E.C., and F.B. performed research; D.M., E.C., and F.B. analyzed data; D.M., F.B., and T.P. wrote the paper.

This work was supported by grants from the Ministero dell'Istruzione, dell'Università e della Ricerca of Italy, and the A. Menarini Industrie Farmaceutiche Riunite Srl (Firenze, Italy).

The authors declare no competing financial interests.

Correspondence should be addressed to Donatella Mutolo, Dipartimento di Scienze Fisiologiche, Università degli Studi di Firenze, Viale G.B. Morgagni 63, 50134 Firenze, Italy. E-mail: donatella.mutolo@unifi.it.

DOI:10.1523/JNEUROSCI.2764-11.2011

Copyright $\odot 2011$ the authors $\quad 0270-6474 / 11 / 3113323-10 \$ 15.00 / 0$
}

verged from the main vertebrate line around 450 million years ago (Hotton, 1976) and proved to be highly useful to provide insights into the basic mechanisms of the central pattern generators (CPG) (Grillner, 2006) and to demonstrate that some of their functional characteristics are retained throughout evolution (Mutolo et al., 2010). Endogenously released excitatory amino acids, but not GABA and glycine, play a crucial role in the lamprey respiratory rhythmogenesis (Bongianni et al., 1999, 2006). In addition, we have suggested (Mutolo et al., 2007, 2010) that a region rostrolateral to the trigeminal motor nucleus, called the paratrigeminal respiratory group (pTRG), has an important function in respiratory rhythm generation corresponding to that of the preBötC in mammals.

Unexpectedly, preliminary experiments performed on lampreys in an attempt to ascertain the presence of respiratory neurons with pacemaker properties (Tryba et al., 2003; Peña et al., 2004; Del Negro et al., 2005) by blocking ionotropic glutamate (Glu) receptors (iGluRs) as well as $\mathrm{GABA}_{\mathrm{A}}$ and glycine receptors showed that respiratory rhythmic activity, although at reduced frequency, persisted. This finding prompted us to further investigate this intriguing issue. Conceivably, other neurotransmitters with an excitatory function on respiratory activity, including ACh (Bianchi et al., 1995; Doi and Ramirez, 2008), could be involved in maintaining the respiratory rhythm.

The present study was carried out on in vitro brainstem preparations of adult lampreys to investigate whether ACh affects respiratory activity possibly through an action on pTRG neurons 
and to identify the AChRs involved. Since one of the main outcome of this study was that endogenous ACh exerts excitatory influences on respiratory activity by acting on $\alpha 7 \mathrm{nAChRs} \mathrm{located}$ within the pTRG, an attempt was also made to test the hypothesis that ACh or other neurotransmitters maintain the respiratory rhythm during blockade of iGlu, $\mathrm{GABA}_{\mathrm{A}}$, and glycine receptors. The effectiveness of iGluR blockade was also investigated in a few experiments.

\section{Materials and Methods}

Lamprey brainstem preparation. This study concerns 87 successful experiments performed on young adult lampreys (Petromyzon marinus). All animal care and experimental procedures were conducted in accordance with the Italian legislation and the official regulations of the European Communities Council on the use of laboratory animals (directive 86/ 609/EEC). The study was approved by the Animal Care and Use Committee of the University of Florence. All efforts were made to minimize the number of animals used. Animal preparation and experimental procedures were similar to those described in previous reports (Bongianni et al., 1999, 2002, 2006; Mutolo et al., 2007, 2010). The animals were anesthetized with tricaine methanesulphonate (100 $\mathrm{mg} \mathrm{l}^{-1}$; MS 222; SigmaAldrich) and transected below the gills. Muscles and connective tissues were removed, and the isolated brain-spinal cord was mounted dorsal side up in a Sylgard-lined recording chamber continuously perfused with a cold solution using a peristaltic pump. The chamber volume was $3.0 \mathrm{ml}$, and the perfusion rate was set at $2.5 \mathrm{ml} \mathrm{min}^{-1}$. Bath temperature was maintained at $9-10^{\circ} \mathrm{C}$. The solution (control solution) flowed from a reservoir and contained the following (in $\mathrm{mm}$ ): $91 \mathrm{NaCl}, 2.1 \mathrm{KCl}, 2.6$, $\mathrm{CaCl}_{2}, 1.8 \mathrm{MgCl}_{2}, 4$ glucose, $23 \mathrm{NaHCO}_{3}$. The solution was continuously bubbled with $95 \% \mathrm{O}_{2} / 5 \% \mathrm{CO}_{2}$ to oxygenate and maintain the $\mathrm{pH}$ in the bath at 7.4. The brain was exposed dorsally and the choroid plexus removed; the brain tissue rostral to the optic tectum was cut and removed. Caudally, a transection was made below the obex, maintaining a minimum length of spinal cord to hold the brainstem preparation. The isolated brainstem of the adult lamprey spontaneously generates respiratory neuronal activity in vitro (fictive respiration); this activity closely resembles that underlying the respiratory behavior of intact animals (Rovainen, 1977, 1983; Thompson, 1985; Russell, 1986; Bongianni et al., 1999, 2002, 2006; Mutolo et al., 2007, 2010). The vast majority of respiratory motoneurons are located in the facial, glossopharyngeal, and especially vagal nuclei (Rovainen, 1974, 1977, 1979; Guimond et al., 2003), while respiration-related neurons possibly responsible for respiratory rhythm generation have been encountered within the pTRG (Mutolo et al., 2007, 2010). Recordings and microinjections within the pTRG as well as neighboring sites were performed under microscope control (Stemi 2000; Zeiss). These maneuvers were facilitated by cutting the roof of the isthmic region along the midline, spreading the alar plates laterally, and pinning them down. In agreement with previous reports (Mutolo et al., 2007,2010 ), these procedures had no significant effect on respiratory activity.

Recording procedures. Efferent respiratory activity was recorded bilaterally from the vagal nerves by means of suction electrodes. The signals were amplified, full-wave rectified, and integrated (low-pass filter; time constant, $10 \mathrm{~ms}$ ). In control trials, brainstem preparations spontaneously produced a stable and regular fictive respiratory rhythm for at least $12 \mathrm{~h}$ (Thompson, 1985; Bongianni et al., 1999, 2002, 2006; Mutolo et al., 2007, 2010). In the present experiments, under control conditions respiratory frequency ranged from 54 to 72 cycles $\mathrm{min}^{-1}$, while the duration of vagal bursts ranged from 21 to $36 \mathrm{~ms}$. Extracellular neuronal activity was recorded with fine (shaft diameter, $0.1 \mathrm{~mm}$ ) tungsten microelectrodes (5 $\mathrm{M} \Omega$ impedance at $1 \mathrm{kHz}$ ) and processed in the same way as vagal activity. The obex was used as a standard point of anatomical reference to evaluate coordinates of recording and microinjection sites. Neuronal activity was recorded from vagal motoneurons $(0.4-0.6 \mathrm{~mm}$ rostral to the obex, $0.3-0.4 \mathrm{~mm}$ lateral to the midline, and $0.15-0.25 \mathrm{~mm}$ below the dorsal surface of the rhombencephalon) as well as from respiration-related neurons of the pTRG $(1.8-2.0 \mathrm{~mm}$ rostral to the obex, $0.8-1.0 \mathrm{~mm}$ lateral to the midline, and $0.25-0.3 \mathrm{~mm}$ below the dorsal surface of the rhomben- cephalon). All of the raw and integrated signals were acquired and analyzed by a personal computer equipped with an analog-to-digital interface (sampling rate $50 \mathrm{kHz}$; Digidata 1200; Molecular Devices) and appropriate software (Axoscope; Molecular Devices) was used. Off-line analysis was performed using Clampfit software (Molecular Devices).

Electrical stimulation. Tungsten microelectrodes $(0.5 \mathrm{M} \Omega$ impedance at $1 \mathrm{kHz}$ ) were used for bipolar electrical stimulation at midline sites rostral to the trigeminal motor nuclei (see Fig. $2 \mathrm{~A}$ ). According to a previous study (Rovainen, 1985), electrical stimulation at these rostral midline sites elicited bursts in the vagal roots through a monosynaptic excitatory pathway. Bipolar stimulation was chosen to minimize the spread of current to neighboring structures. Electrode tips were placed $\sim 0.3 \mathrm{~mm}$ apart over the midline, at the depth of $\sim 0.1 \mathrm{~mm}$, between the pair of isthmic Müller cells. Stimulations consisted of single stimuli (0.1 ms duration, current rectangular pulses at intensities of 5-12 $\mu \mathrm{A})$. Stimuli were delivered by a constant-current unit driven by a stimulator (BM ST6; Biomedica Mangoni). Current intensity could be read on an oscilloscope by monitoring the voltage drop through a small series resistor. The intensity of stimulation was adjusted to a level capable of inducing maximum evoked responses. Stimuli were delivered between vagal bursts with an interval of $\sim 3 \mathrm{~s}$. Stimulation-evoked responses were recorded from vagal roots and evaluated as superimposed traces.

Drug application. Drugs were either applied through the perfusing solution or microinjected into the PTRG as well as into the region of vagal motoneurons. In each experiment, the preparation was perfused with the control solution for at least $60 \mathrm{~min}$ before control recordings. Unless stated otherwise, only one drug was tested in each preparation. The following drugs were used: $100 \mu \mathrm{M}$ to $1 \mathrm{~mm}$ physostigmine (an acetylcholinesterase inhibitor; Sigma-Aldrich), 10-50 $\mu$ m pilocarpine hydrochloride (a mAChR agonist; Tocris Bioscience), $10 \mu \mathrm{M}$ atropine sulfate salt (a nonselective $\mathrm{mAChR}$ antagonist; Sigma-Aldrich), $1 \mu \mathrm{M}$ to $1 \mathrm{~mm}$ nicotine hydrogen tartrate salt (an nAChR agonist, Sigma-Aldrich), $100 \mu \mathrm{m}$ to $1 \mathrm{~mm}$ D-tubocurarine chloride hydrate (a non-selective $\mathrm{nAChR}$ antagonist, Sigma-Aldrich), 0.25-2.5 $\mu \mathrm{M} \alpha$-bungarotoxin (an $\alpha 7$ selective nAChR antagonist; SigmaAldrich), $10 \mu \mathrm{M}$ dihydro- $\beta$-erythroidine hydrobromide (an $\alpha 4 \beta 2$ selective $\mathrm{nAChR}$ antagonist; Sigma-Aldrich), $10 \mu \mathrm{m}$ hexamethonium chloride (a preferentially blocker of $n A C h R s$ at autonomic ganglia; Sigma-Aldrich), 100 $\mu \mathrm{M}$ suramin sodium salt and $100 \mu \mathrm{M}$ pyridoxal-phosphate-6-azophenyl$2^{\prime}, 4^{\prime}$-disulfonic acid tetrasodium salt (PPADS; broad-spectrum antagonists at P2X and P2Y purinergic receptors; Sigma-Aldrich), $500 \mu \mathrm{M}(R S)-\alpha-$ methyl-4-carboxyphenylglycine disodium salt (MCPG; a nonselective group I/group II metabotropic GluR antagonist; Tocris Bioscience), $10 \mu \mathrm{M}$ methysergide maleate (a nonselective 5-HT receptor antagonist; Tocris Bioscience), $10 \mu \mathrm{M}$ CP-99,994 (an NK1 receptor antagonist; gift from Pfizer), 10 $\mu \mathrm{M}$ MEN 10376 (an NK2 receptor antagonist; Tocris Bioscience), and $10 \mu \mathrm{M}$ SB 222200 (an NK3 receptor antagonist; Tocris Bioscience). In addition, the following antagonists of fast excitatory and inhibitory transmission were used: $100 \mu \mathrm{M} \mathrm{D}-(-)-2$-amino-5-phosphonopentanoic acid (D-AP5; an NMDA receptor antagonist; Tocris Bioscience), 20-100 $\mu \mathrm{M}$ 6-cyano-7nitroquinoxaline-2,3-dione (CNQX; a nonNMDA receptor antagonist; Tocris Bioscience), $10 \mu \mathrm{m}$ bicuculline methiodide (a GABA receptor antagonist; Sigma-Aldrich), and $10 \mu \mathrm{M}$ strychnine (a glycine receptor antagonist; Tocris Bioscience). The drug concentrations and the application time were similar to those used in previous studies (Bongianni et al., 1999, 2002, 2006; Aberger et al., 2001; Le Ray et al., 2003, 2004; Shao and Feldman, 2002; Tryba et al., 2003; Peña et al., 2004; Del Negro et al., 2005; Lorier et al., 2007; Shao et al., 2008; St. John and Leiter, 2008; Mutolo et al., 2010). Unless stated otherwise, the brainstem was perfused with each drug for $30 \mathrm{~min}$; after completion of each drug challenge, the preparation was allowed to recover by perfusing it with the control solution. All drugs used, except for pilocarpine, were dissolved in distilled water made up to a stock solution and stored as small aliquots in a freezer until use. Pilocarpine was dissolved in dimethyl sulfoxide (DMSO; Sigma-Aldrich) to produce stock solutions. Stock solutions were diluted in the perfusing solution to the final desired concentration immediately before application. Control experiments, performed by adding equivalent amounts of DMSO in the perfusing solution, did not produce respiratory effects. In some experiments, we also ascertained the effectiveness of nAChR blockade induced by $100 \mu \mathrm{M}$ D-tubocurarine or $0.25 \mu \mathrm{M} \alpha$-bun- 
A Control

VA
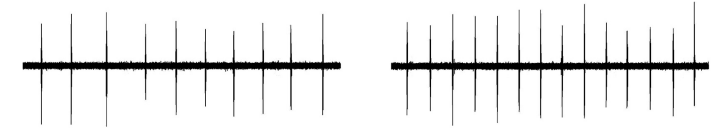
$100 \mu \mathrm{M}$ Physo bath application

IVA

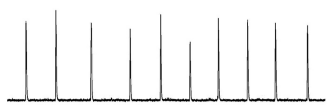

B

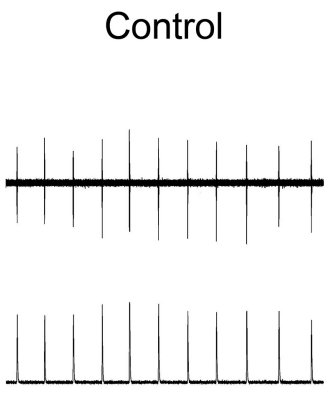

C Control
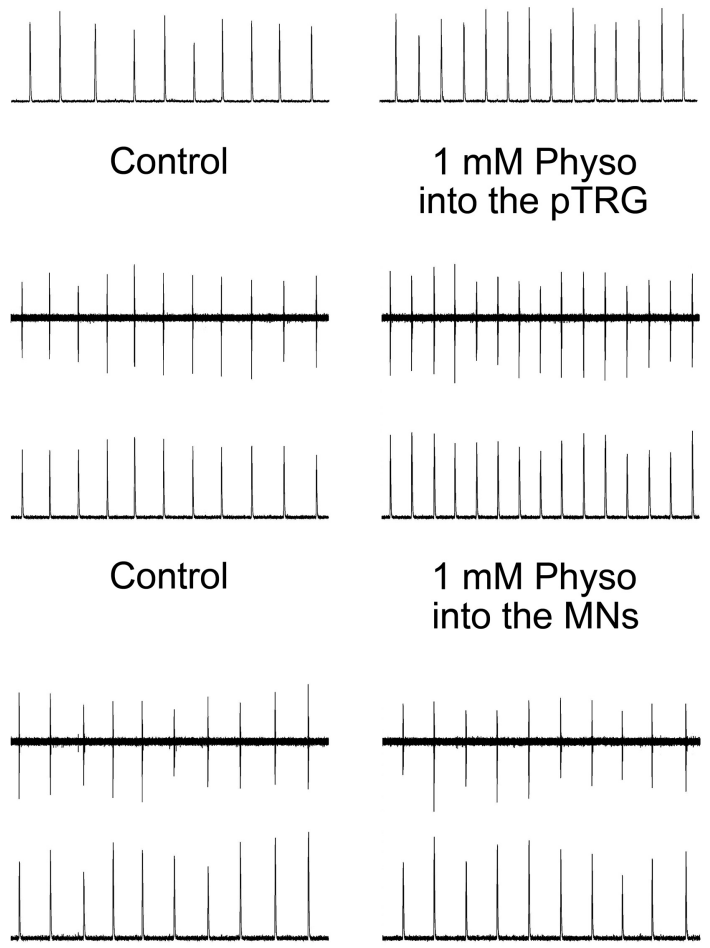

$1 \mathrm{mM}$ Physo into the pTRG

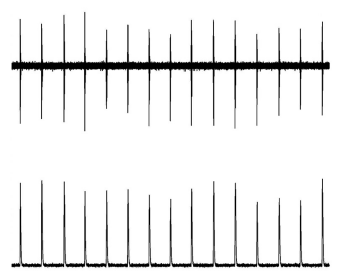

$1 \mathrm{mM}$ Physo into the MNs

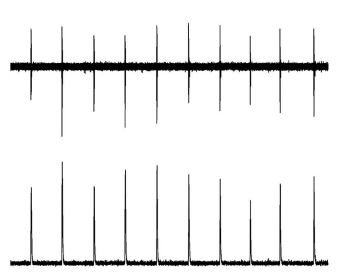

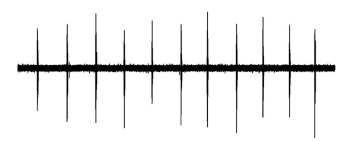
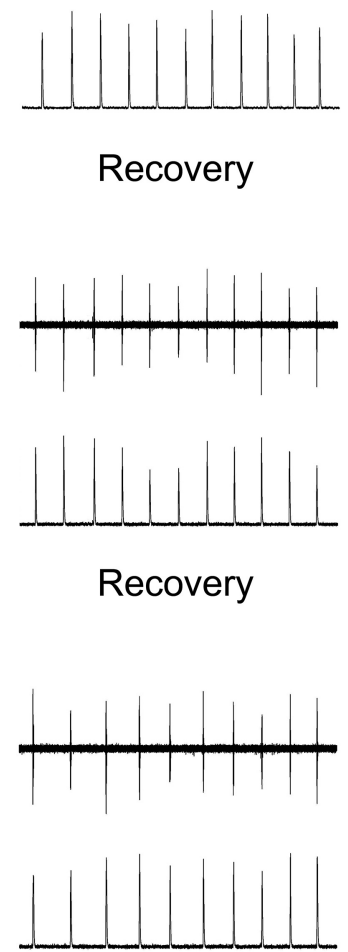

Figure 1. Examples of respiratory responses caused by the acetylcholinesterase inhibitor physostigmine (Physo). $A, B$, Increases in respiratory frequency $\sim 15 \mathrm{~min}$ after bath application of $100 \mu$ m physostigmine $(\boldsymbol{A})$ and $\sim 5 \mathrm{~min}$ after bilateral microinjections of $1 \mathrm{~mm}$ physostigmine into the PTRG (B). $\boldsymbol{C}$, No changes in respiratory activity $\sim 5$ min following bilateral microinjections of $1 \mathrm{~mm}$ physostigmine into the region of vagal motoneurons (MNs). VA, Raw vagal nerve activity; IVA, integrated vagal nerve activity.

garotoxin. First, the antagonist was applied alone for $30 \mathrm{~min}$, and subsequently $1 \mu \mathrm{M}$ nicotine was added and maintained in the bath for $20 \mathrm{~min}$.

Bilateral microinjections (0.5-1 nl) of physostigmine (1 mM), nicotine (1 mM), D-tubocurarine ( $1 \mathrm{mM})$, and $\alpha$-bungarotoxin $(2.5 \mu \mathrm{M})$ were performed by means of glass micropipettes (tip diameter, $10-15 \mu \mathrm{m}$ ) and by applying pressure pulses of $50-100 \mathrm{~ms}$ with a Picospritzer (General Valve) connected to the injection pipette. The injected volume was estimated by measuring the diameter of a droplet ejected from the tip of the pipette. Bilateral microinjections were performed using a single micropipette that was withdrawn after the first microinjection and then introduced contralaterally for the second injection. The interval between the two microinjections was $\leq 20 \mathrm{~s}$. Bilateral microinjections were performed into the PTRG and the region of vagal motoneurons on the basis of both extracellular recordings and coordinates. The concentrations of microinjected drugs were selected in preliminary trials and were in the same range as those used in previous microinjection studies (Aberger et al., 2001; Le Ray et al., 2003). For further considerations on the concentrations of neuroactive drugs, see Mutolo et al. (2007). The inactive dye Fast Green (0.2\%; Sigma-Aldrich) was added to the drug solution to visually assess the spread and the approximate localization of the injectate. The microinjections were associated with the appearance of dye spots (diameter $\sim 0.2 \mathrm{~mm}$ ) confined to the investigated regions (Mutolo et al., 2007). Localization of the pTRG was judged on the basis of dye spot position with respect to the sulcus limitans of His. The depth of the injectate was inferred from that of rhythmic extracellular neuronal activ-
Recovery

Recovery

$\overline{1 \mathrm{~s}}$

ity recorded previously. Control microinjections of the perfusing solution with Fast Green were also made. On some occasions $(n=3)$, the locations of the recording sites within the pTRG were marked by injecting the perfusing solution (1 nl) containing 10\% horseradish peroxidase (HRP; Sigma-Aldrich) according to a technique described in previous studies (Brocard et al., 2005; Mutolo et al., 2007). The localization of the injections performed into the vagal motor nucleus was evaluated only by the associated dye spots according to the procedure described above for the pTRG.

A cocktail of antagonists for NMDA receptors (100 $\mu \mathrm{M}$ D-AP5), non-NMDA receptors $(20 \mu \mathrm{M}$ $\mathrm{CNQX}), \mathrm{GABA}_{\mathrm{A}}$ receptors (10 $\mu \mathrm{m}$ bicuculline), and glycine receptors (10 $\mu \mathrm{M}$ strychnine) was applied to lamprey brainstem preparations through the perfusing solution. During cocktail perfusion, some drugs could either be added to the bath or microinjected into the pTRG. The preparation was then allowed to recover by perfusing it with the cocktail solution. On some occasions, CNQX and D-AP5 were applied first to abolish the respiratory rhythm (Bongianni et al., 1999), and the other components of the cocktail solution were added subsequently. In additional experiments, blockade of iGluRs was evaluated by stimulation-evoked responses both during application of iGluR antagonists alone and following the subsequent application of bicuculline and strychnine. In each experiment, after blockade of iGlu, $\mathrm{GABA}_{\mathrm{A}}$, and glycine receptors, the preparation was maintained under these conditions for $\sim 60$ min and thereafter was allowed to recover by perfusing it with the control solution.

Histology. The fixed brains ( $4 \%$ paraformaldehyde in $0.1 \mathrm{~m}$ phosphate buffer, $\mathrm{pH} 7.4$, overnight) were cryoprotected with $30 \%$ sucrose, frozen, and cut at $25 \mu \mathrm{m}$ thickness on a cryostat. Coronal sections stained with cresyl violet were used for the histological analysis. To reveal the presence of HRP, the sections were reacted with $3,3^{\prime}$-diaminobenzidine tetrahydrochloride $(0.05 \%)$ and $\mathrm{H}_{2} \mathrm{O}_{2}(0.01 \%)$ in PBS for $5 \mathrm{~min}$ and gently counterstained with cresyl violet according to a technique described previously (Mutolo et al., 2007).

Data analysis. Respiratory frequency (cycles per minute; cycles $\min ^{-1}$ ), vagal burst duration (milliseconds; measured on raw activity), and peak amplitude of integrated vagal activity (taken as a reliable index of the intensity of vagal bursts; arbitrary units) were measured and averaged for $20 \mathrm{~s}$ both in the period immediately preceding each trial (control values) and, unless stated otherwise, at 2 min intervals during drug application. Average values of respiratory variables observed in control conditions and at the time when the maximum response occurred were considered for statistical analysis (SigmaStat; Jandel Scientific Software). Paired $t$ tests were used to evaluate changes in respiratory variables induced by each drug added to the bath. The same statistical analysis was used to evaluate changes in respiratory variables induced by each agonist in the presence of the specific antagonist and to assess the effects of drug microinjections. In microinjection trials, respiratory variables were measured and averaged for $20 \mathrm{~s}$ immediately before the microinjections, as well as at the time when the maximum response occurred. Changes in respiratory variables were also expressed as percentage variations of control values. The number of preparations used in each set of drug challenges is indicated by $n$. The number of trials has been reported on some occasions to imply that some of them were executed in a single preparation. All values 
are presented as means \pm SEM, and $p<0.05$ was considered as significant.

\section{Results}

Respiratory role of $\mathrm{ACh}$

A total number of six preparations was used to investigate the respiratory responses to physostigmine. Bath application of $100 \mu \mathrm{M}$ physostigmine induced marked increases in respiratory frequency from $65.1 \pm 4.7$ to $89.3 \pm 6.5$ cycles $\min ^{-1}(37.2 \pm 2.2 \% ; n=$ $6 ; p<0.01)$ without significant changes in peak amplitude and duration of vagal bursts (Fig. 1A). Increases in respiratory frequency started within $\sim 5$ min after bath application, developed progressively, and reached a maximum within $\sim 15$ min. Respiratory activity recovered within 60 min washout. In the same preparations, we performed bilateral microinjections into the pTRG or the vagal motoneuron region on the basis of both extracellular recordings and coordinates. Bilateral microinjections of physostigmine (1 mM; $0.5-1 \mathrm{pmol}$ ) into the pTRG increased respiratory frequency from $61.1 \pm 2.5$ to $82.8 \pm 3.4$ cycles $\min ^{-1}(36.1 \pm 7.3 \% ; n=4 ; p<0.01)$ without significant changes in peak amplitude and duration of vagal bursts (Fig. $1 B)$. The respiratory responses started within 1 min after the injections and reached a maximum within $\sim 5 \mathrm{~min}$. Respiratory activity recovered gradually within 25 min after the injections. Bilateral microinjections of physostigmine (1 $\mathrm{mM}$; $0.5-1 \mathrm{pmol})$ into the region of vagal motoneurons $(n=3)$ did not cause any obvious effect (Fig. 1C). The localization of injections sites is illustrated in Figure 2. An histological control of an HRP injection site is also shown. The localization of the injected area closely corresponds to that already reported in a previous study (Mutolo et al., 2007). Control microinjections of equal volumes of the vehicle solution containing $0.2 \%$ Fast Green dye at responsive sites (three trials) as well as bilateral microinjections of $1 \mathrm{~mm}$ physostigmine at neighboring sites $0.4 \mathrm{~mm}$ or more away from the responsive region (four trials) did not cause any obvious respiratory response.

To identify the receptors involved in ACh-mediated effects, specific agonists and antagonists acting on nAChRs or $\mathrm{mAChRs}$ were used. Bath application of $1 \mu \mathrm{M}$ nicotine $(n=5)$ increased respiratory frequency from $65.7 \pm 4.2$ to $91.1 \pm 3.4$ cycles $\min ^{-1}$ (39.7 $\pm 5.2 \%$; $p<0.001)$. Peak amplitude and duration of vagal bursts did not vary. These responses started within 2 min after bath application, developed progressively, and reached a maximum within $\sim 10 \mathrm{~min}$. Respiratory activity recovered within $60 \mathrm{~min}$

$\mathrm{D}$
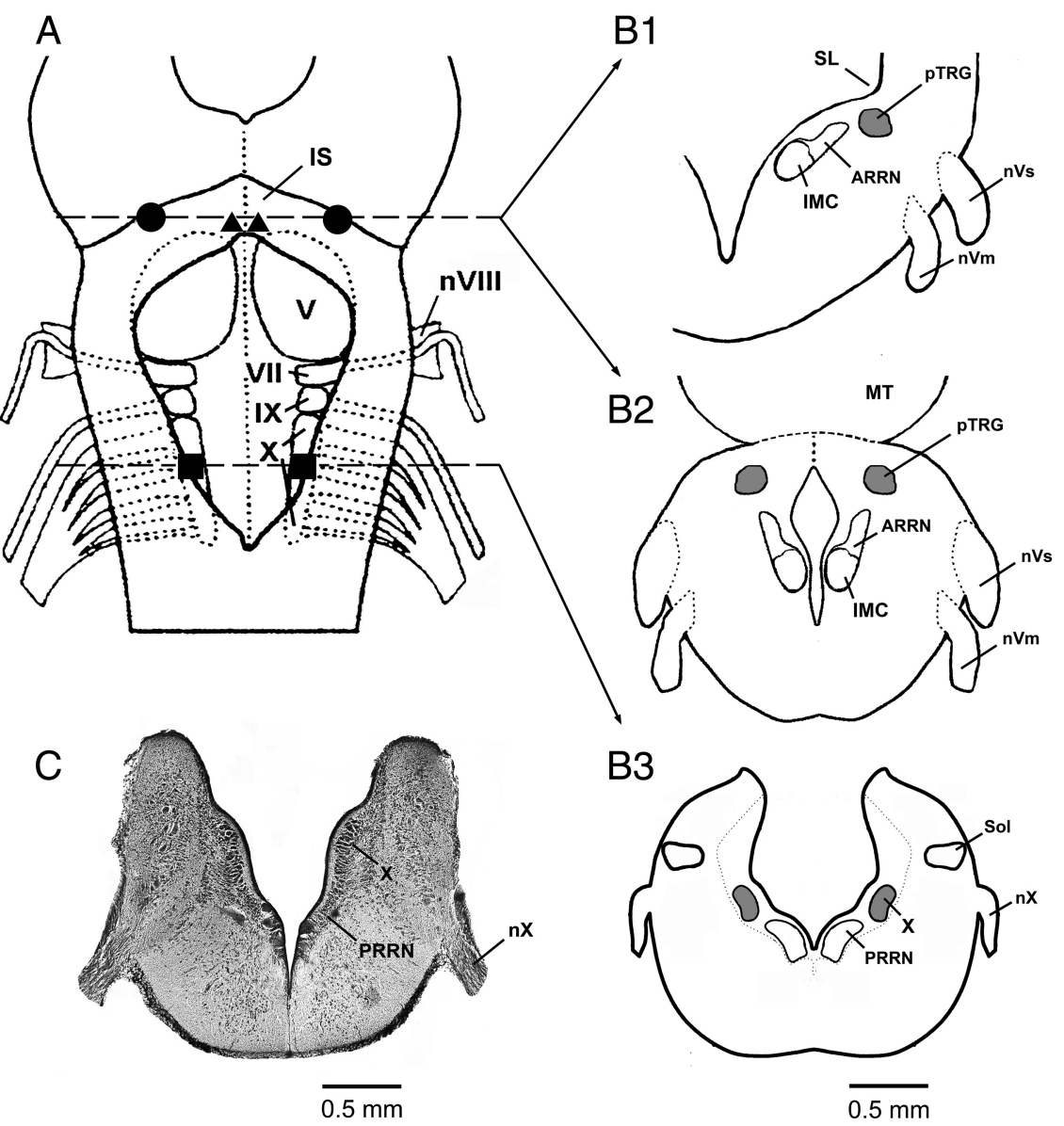

$E$

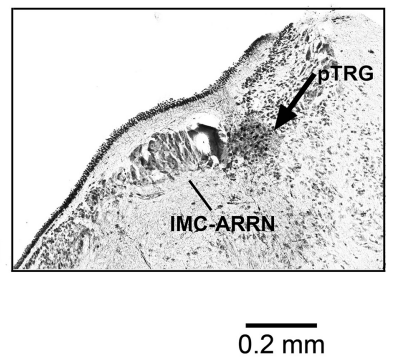

Figure 2. Localization of recording and injection sites. $A$, Diagrammatic illustration of a dorsal view of the lamprey rhombencephalon showing the localization of the pTRG $(\mathbf{O})$ and the vagal motor nucleus $(\square)$. Sites of bipolar electrical stimulation arealso indicated $(\boldsymbol{\Delta}) \cdot \mathbf{B}$, $B 2$, Schematic drawings of transverse sections of the rostral rhombencephalon at the samelevel as indicated in $\boldsymbol{A}$ (dashed line) showing the location of the PTRG (shaded areas). The outlines of the map reported in $\mathbf{B} 1$ are derived from a selected section of one histological preparation (camera lucida redrawing) of the brainstem. In this case, unlike the transverse section in $\boldsymbol{B 2}$, the roof of the isthmic region was cut along the midline, and the alar plates were spread laterally and pinned down to perform recordings and microinjections within the pTRG. $\boldsymbol{B}$, Diagram of a transverse section of the caudal rhombencephalon at the level indicated in $\boldsymbol{A}$ (dashed line) showing the location of the vagal motor nucleus (shaded areas). The outlines of schematic drawings in $\mathbf{B 2}$ and $\mathbf{B} 3$ are derived from Auclair et al. (2004). $\boldsymbol{C}, \boldsymbol{D}$, Photomicrographs of transverse sections already schematically illustrated in $\boldsymbol{B}$ and $\boldsymbol{B}$ 1 showing the location of the vagal motor nucleus and the PTRG (outlined by the dotted line), respectively. $E$, Photomicrograph at higher magnification of the portion of a transverse section approximately indicated by the rectangle in $\boldsymbol{D}$, showing the location of an HRP injectate into the PTRG (arrow) of a different preparation. ARRN, Anterior rhombencephalic reticularnucleus; IMC, isthmic Müller cell; IS, isthmus; $M T$, mesencephalictectum; $n V m$, motor root of the trigeminal nerve; $n V s$, sensory root of the trigeminal nerve; nVIII, vestibular nerve; $n X$, vagal nerve; PRRN, posterior rhombencephalic reticular nucleus; PTRG, paratrigeminal respiratory group; SL, sulcus limitans of His; Sol, nucleus of the solitary tract; $\mathrm{V}$, trigeminal motor nucleus; VII, facial motor nucleus; IX, glossopharyngeal motor nucleus; $\mathrm{X}$, vagal motor nucleus.

washout. In four preparations, bath application of pilocarpine at $10 \mu \mathrm{M}$, or even at $50 \mu \mathrm{M}$ in two cases, did not alter respiratory activity. Similarly, bath application of $10 \mu \mathrm{M}$ atropine $(n=4)$ did not affect the respiratory motor output. These respiratory effects are illustrated in Figure $3 \mathrm{~A}$. 

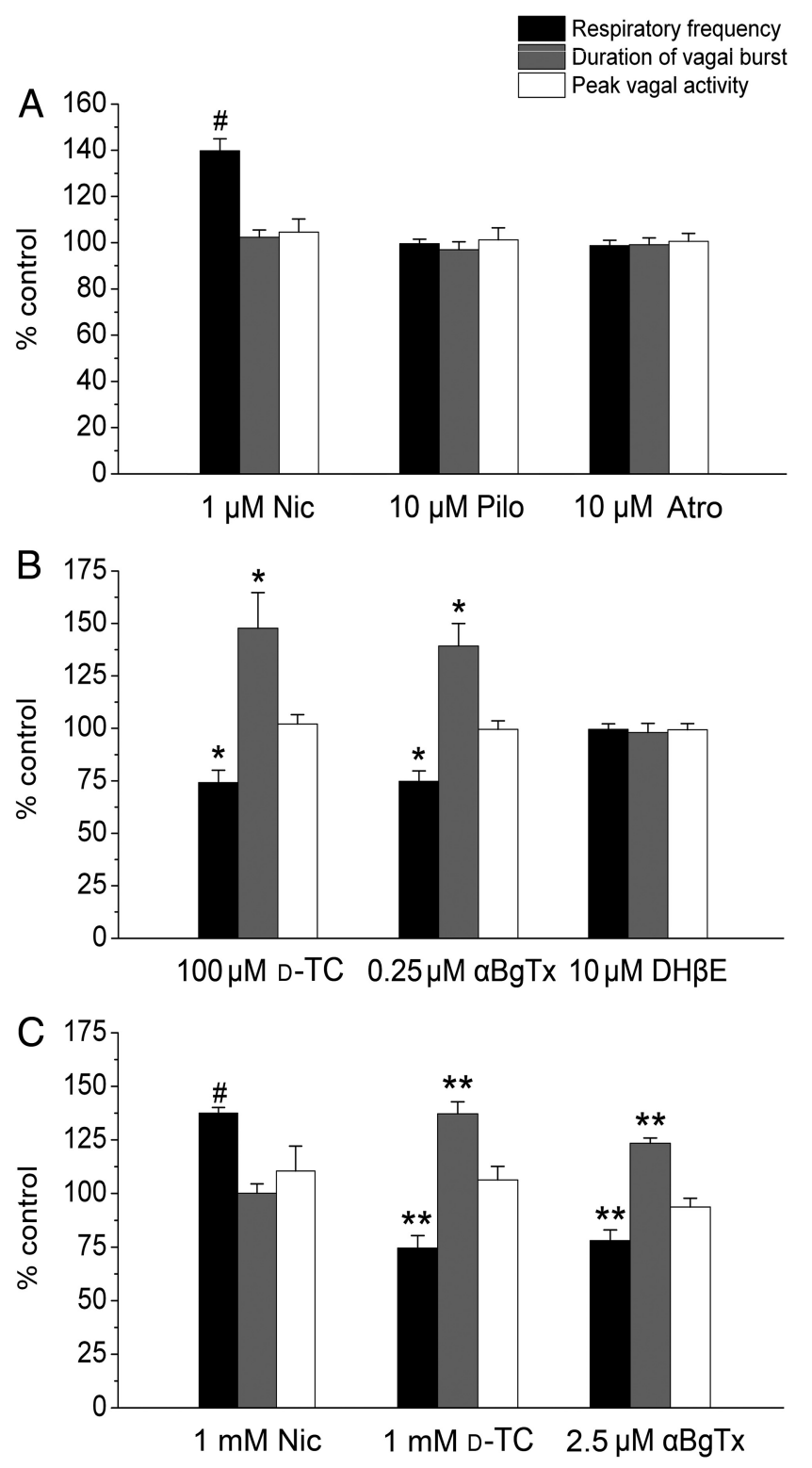

Figure 3. Respiratory responses to nicotinic and muscarinic receptor agonists and antagonists. $A$, Histograms illustrating maximum changes in respiratory frequency, vagal burst duration, and peak vagal activity induced by bath application of $1 \mu \mathrm{m}$ nicotine (Nic; $n=5), 10 \mu \mathrm{m}$ pilocarpine (Pilo; $n=4)$, and $10 \mu \mathrm{m}$ atropine (Atro; $n=4)$. B, Histograms showing maximum changes in respiratory frequency, vagal burst duration, and peak vagal activity induced by 100 $\mu \mathrm{M}$ D-tubocurarine (D-TC; $n=6), 0.25 \mu \mathrm{M} \alpha$-bungarotoxin $(\alpha \mathrm{BgTx} ; n=6)$, and $10 \mu \mathrm{M}$ dihydro- $\beta$-erythroidine ( $\mathrm{DH} \beta \mathrm{E} ; n=4)$. C, Histograms showing maximum changes in respiratory frequency, vagal burst duration, and peak vagal activity induced by $1 \mathrm{~mm} \mathrm{Nic}(n=6), 1 \mathrm{~mm}$ D-TC $(n=6)$, and $2.5 \mu \mathrm{m} \alpha \operatorname{BgTx}(n=6)$ microinjected into the pTRG. Values are means \pm SEM. ${ }^{*} p<0.05 ;{ }^{* *} p<0.01 ;{ }^{\#} p<0.001$.

To test whether nAChRs are involved in the control of baseline respiratory activity, we investigated the effects of various $\mathrm{nAChR}$ antagonists. Bath application of $100 \mu \mathrm{M}$ D-tubocurarine $(n=6)$ caused reductions in respiratory frequency from $63.5 \pm$ 3.6 to $47.8 \pm 3.3$ cycles $\min ^{-1}(25.8 \pm 6.0 \% ; p<0.05)$ and increases in the duration of vagal bursts from $25.6 \pm 1.3$ to $38.0 \pm 3.5 \mathrm{~ms}(49.1 \pm 15.0 \% ; p<0.05)$ without significant changes in their peak amplitude. Bath application of $0.25 \mu \mathrm{M}$ $\alpha$-bungarotoxin $(n=6)$ provoked decreases in respiratory fre-

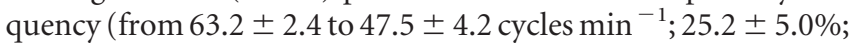
$p<0.05$ ) and increases in the duration of vagal bursts (from
$27.9 \pm 1.7$ to $38.8 \pm 3.5 \mathrm{~ms} ; 39.8 \pm 10.6 \% ; p<0.05)$ without variations in their peak amplitude. In some of these preparations, the presence of $100 \mu \mathrm{M}$ D-tubocurarine $(n=2)$ or $0.25 \mu \mathrm{M}$ $\alpha$-bungarotoxin $(n=2)$ in the bath proved to prevent the excitatory effects on respiration induced by the application of $1 \mu \mathrm{M}$ nicotine (data not shown). On the contrary, no respiratory effects were observed following bath application of $10 \mu \mathrm{M}$ dihydro- $\beta$ erythroidine $(n=4)$ or $10 \mu \mathrm{M}$ hexamethonium $(n=3$; data not shown). Histograms in Figure $3 B$ illustrate changes in respiratory frequency, duration, and peak amplitude of vagal bursts induced by D-tubocurarine, $\alpha$-bungarotoxin, and dihydro- $\beta$-erythroidine. The respiratory responses started within $\sim 5$ min after bath application of D-tubocurarine or $\alpha$-bungarotoxin developed gradually and reached a maximum within $\sim 15 \mathrm{~min}$. Respiratory activity recovered within $60 \mathrm{~min}$ washout.

\section{Nicotinic receptors within the pTRG}

Respiratory responses induced by bilateral microinjections of nicotine, D-tubocurarine, and $\alpha$-bungarotoxin into the pTRG have been reported in Figure $3 C$. Microinjections of $1 \mathrm{~mm}$ nicotine $(0.5-1 \mathrm{pmol} ; n=6)$ caused significant increases in respiratory frequency from $64.2 \pm 3.4$ to $88.5 \pm 5.6$ cycles $\mathrm{min}^{-1}$ $(37.3 \pm 2.6 \% ; p<0.001)$ without significant changes in the duration and amplitude of vagal bursts. Microinjections of $1 \mathrm{~mm}$ D-tubocurarine $(0.5-1 \mathrm{pmol} ; n=6)$ caused decreases in respiratory frequency from $67.1 \pm 3.4$ to $49.7 \pm 4.5$ cycles $\mathrm{min}^{-1}$ $(25.7 \pm 5.8 \% ; p<0.01)$ and increases in vagal burst duration from $26.2 \pm 1.4$ to $35.7 \pm 2.0 \mathrm{~ms}(36.7 \pm 5.6 \% ; p<0.01)$ without changes in their peak amplitude. Similarly, microinjections of 2.5 $\mu \mathrm{M} \alpha$-bungarotoxin ( $1.25-2.5 \mathrm{fmol} ; n=6)$ decreased respiratory frequency (from $62.5 \pm 3.1$ to $48.6 \pm 3.4$ cycles min $^{-1} ; 22.3 \pm$ $5.0 \% ; p<0.01$ ) and increased the duration of vagal bursts (from $28.9 \pm 1.7$ to $35.0 \pm 2.3 \mathrm{~ms} ; 22.9 \pm 2.5 \% ; p<0.01)$ without changes in their peak amplitude. All these respiratory responses started within 1 min and reached a maximum within $\sim 5 \mathrm{~min}$. Respiratory activity recovered gradually within 60 min after the injections. Bilateral microinjections at neighboring sites $0.4 \mathrm{~mm}$ or more away from the responsive region (nicotine, four trials; D-tubocurarine, six trials; $\alpha$-bungarotoxin, four trials) did not cause any respiratory change. Bilateral microinjections of nicotine $(n=2)$, D-tubocurarine $(n=3)$, and $\alpha$-bungarotoxin $(n=$ 2) performed in the same preparations into the region where vagal motoneurons are located did not alter respiratory activity (data not shown).

\section{Blockade of iGlu, $\mathrm{GABA}_{\mathrm{A}}$, and glycine receptors}

A cocktail of antagonists for NMDA (100 $\mu \mathrm{M}$ D-AP5), nonNMDA (20 $\mu \mathrm{M}$ CNQX), GABA ( $10 \mu \mathrm{M}$ bicuculline), and glycine (10 $\mu \mathrm{M}$ strychnine) receptors was applied through the perfusing solution (Tryba et al., 2003; Peña et al., 2004; Del Negro et al., 2005). Respiratory activity was not abolished. The analysis of cumulative data from preparations in which blockade of these receptors was performed $(n=27)$ showed that respiratory frequency decreased from $66.1 \pm 2.1$ to $36.1 \pm 1.3$ cycles $\mathrm{min}^{-1}$ (44.3 $\pm 2.6 \% ; p<0.001)$, whereas the duration of vagal bursts increased from $28.6 \pm 0.5$ to $59.9 \pm 2.3 \mathrm{~ms}(109.5 \pm 6.3 \%$; $p<$ 0.001 ), without concomitant changes in their peak amplitude (Fig. 4). The respiratory responses developed progressively and reached a maximum within $\sim 30 \mathrm{~min}$. Respiratory activity recovered within $60 \mathrm{~min}$ washout. In two of these experiments, $20 \mu \mathrm{M}$ CNQX and $100 \mu \mathrm{M}$ D-AP5 were applied first and caused, as expected (Bongianni et al., 1999), the cessation of the respiratory rhythm within $\sim 20 \mathrm{~min}$. Thus, CNQX and D-AP5 applied alone 
provoked a dramatically different response compared with that induced by the cocktail solution. Thereafter, when $10 \mu \mathrm{M}$ bicuculline and $10 \mu \mathrm{M}$ strychnine were added to the bath, respiratory activity after $\sim 1$ min progressively resumed both as amplitude and frequency, and within $\sim 10$ min displayed steady-state characteristics similar to those described above following cocktail application.

Experiments were performed to verify whether respiratory activity could be affected during cocktail perfusion $(>30$ $\mathrm{min}$ ) by the application of receptor antagonists of different neurotransmitters. The brainstem was perfused with each drug for $\sim 30 \mathrm{~min}$. During this period, bath application of the nonselective 5-HT receptor antagonist methysergide $(10 \mu \mathrm{M}$; $n=4$ ), the nonselective group I/group II metabotropic GluR antagonist MCPG (500 $\mu \mathrm{M} ; n=3$ ), and the broad-spectrum antagonists at $\mathrm{P} 2 \mathrm{X}$ and $\mathrm{P} 2 \mathrm{Y}$ purinergic receptors suramin or PPADS $(100 \mu \mathrm{M} ; n=$ 3) did not cause any apparent change in either frequency or amplitude of vagal bursts (Fig. 5A). In the same preparations, after appropriate $(>60$ min) washout in the presence of the cocktail solution, bath application of the NK1 receptor antagonist CP-99,994 (10 $\mu \mathrm{M} ; n=$ 4), the NK2 receptor antagonist MEN $10376(10 \mu \mathrm{M} ; n=3)$, and the NK3 receptor antagonist SB $222200(10 \mu \mathrm{M} ; n=3)$ did not cause any change in the respiratory variables (data not shown). On the contrary, in seven additional preparations, bath application of $0.25 \mu \mathrm{M} \alpha$-bungarotoxin $(n=5)$ or $100 \mu \mathrm{M}$ D-tubocurarine $(n=2)$ abolished respiratory activity within $\sim 10$ $\min$. The respiratory frequency decreased progressively without appreciable changes in peak vagal activity (Fig. 5B). Respiratory activity recovered within $60 \mathrm{~min}$ washout in the presence of the cocktail solution. In three of these preparations, after recovery, bilateral microinjections of $2.5 \mu \mathrm{M} \alpha$-bungarotoxin (1.25-2.5 fmol) into the pTRG caused similar depressant effects on respiratory activity. These responses started within $1 \mathrm{~min}$ and led to the complete suppression of vagal bursts within $\sim 5 \mathrm{~min}$ (Fig. $5 B)$. Rhythmic respiratory activity recovered gradually within 60 min after the injections. Bilateral microinjections performed 0.4 $\mathrm{mm}$ or more away from the responsive region (four trials) did not affect respiratory activity.

In five additional preparations, neurons with respirationrelated activity were recorded within the pTRG both before and after cocktail application (Fig. 6). These neurons maintained rhythmic activity synchronous with vagal bursts at decreased frequency after cocktail application. Under these conditions, rhythmic neuronal and vagal activities displayed an increase in frequency that reached a maximum within $\sim 10 \mathrm{~min}$ in response to bath application of $1 \mu \mathrm{M}$ nicotine $(n=2)$. On the contrary, they were abolished within the same time interval following bath application of $0.25 \mu \mathrm{M} \alpha$-bungarotoxin $(n=3)$.

Since glutamatergic transmission, as a rule, mediates the excitatory input to lamprey motoneurons (for review, see Grillner and Jessell, 2009), the present results raised the problem of how the respiratory drive is conveyed to vagal motoneurons in the presence of iGluR antagonists. We addressed this issue in a few experiments $(n=4)$ by using electrical stimulation of descending axons of premotoneurons at the rostral midline (Rovainen, 1985) and recording the evoked responses from vagal roots. Evoked vagal bursts, albeit at reduced intensity, were recorded both during apnea caused by iGluR antagonists $(20 \mu \mathrm{M}$ CNQX and 100 $\mu \mathrm{M}$ D-AP5, $n=2$ or $100 \mu \mathrm{M}$ CNQX and $100 \mu \mathrm{M} \mathrm{D-AP5,} n=2$ ) and $10 \mathrm{~min}$ after the subsequent application of $10 \mu \mathrm{M}$ bicuculline and $10 \mu \mathrm{M}$ strychnine that caused, as reported above for the lower CNQX concentration, the resumption of rhythmic respiratory activity (data not shown). The reductions in the intensity of evoked vagal bursts evaluated by superimposed sweeps were observed in each of these experiments. These results deserve further investigation to provide a quantitative evaluation of the observed phenomena and lead to a reliable interpretation of them. Nevertheless, they clearly imply that blockade of glutamatergic transmission was not complete, at least at the vagal motoneuron level, but sufficient to arrest respiratory rhythm-generating mechanisms at the pTRG level when iGluR antagonists were applied alone.

Data concerning the role of iGluRs and nAChRs can be included in viable conceptual models of synaptic interactions between neurons of the pTRG. Two models are proposed, one based on neurons that corelease Glu and ACh (corelease hypothesis) (Grillner and Jessell, 2009) and the other based on the presence of separate neurons releasing either Glu or ACh (separate release hypothesis). In both models, neurons have recurrent excitatory actions on other interneurons (Bongianni et al., 1999; Mutolo et al., 2010). Cholinergic neurons are probably located within the pTRG, but an action from cholinergic neurons located elsewhere cannot be ruled out completely (Pombal et al., 2001; Le Ray et al., 2003). Inhibitory neurons synapse on single or several excitatory interneurons (Bongianni et al., 2006). In the first model, removal of inhibition by bicuculline and strychnine may enhance the corelease of Glu and ACh, but mainly ACh could be effective because of the relatively intense impairment of glutamatergic transmission. In the second model, removal of inhibition may increase the release from glutamatergic and/or cholinergic neurons. It seems plausible that also under these circumstances 
A Cocktail: $20 \mu \mathrm{M} \mathrm{CNQX}+100 \mu \mathrm{M}$ D-AP5 + $10 \mu \mathrm{M}$ Bic $+10 \mu \mathrm{M}$ Stryc
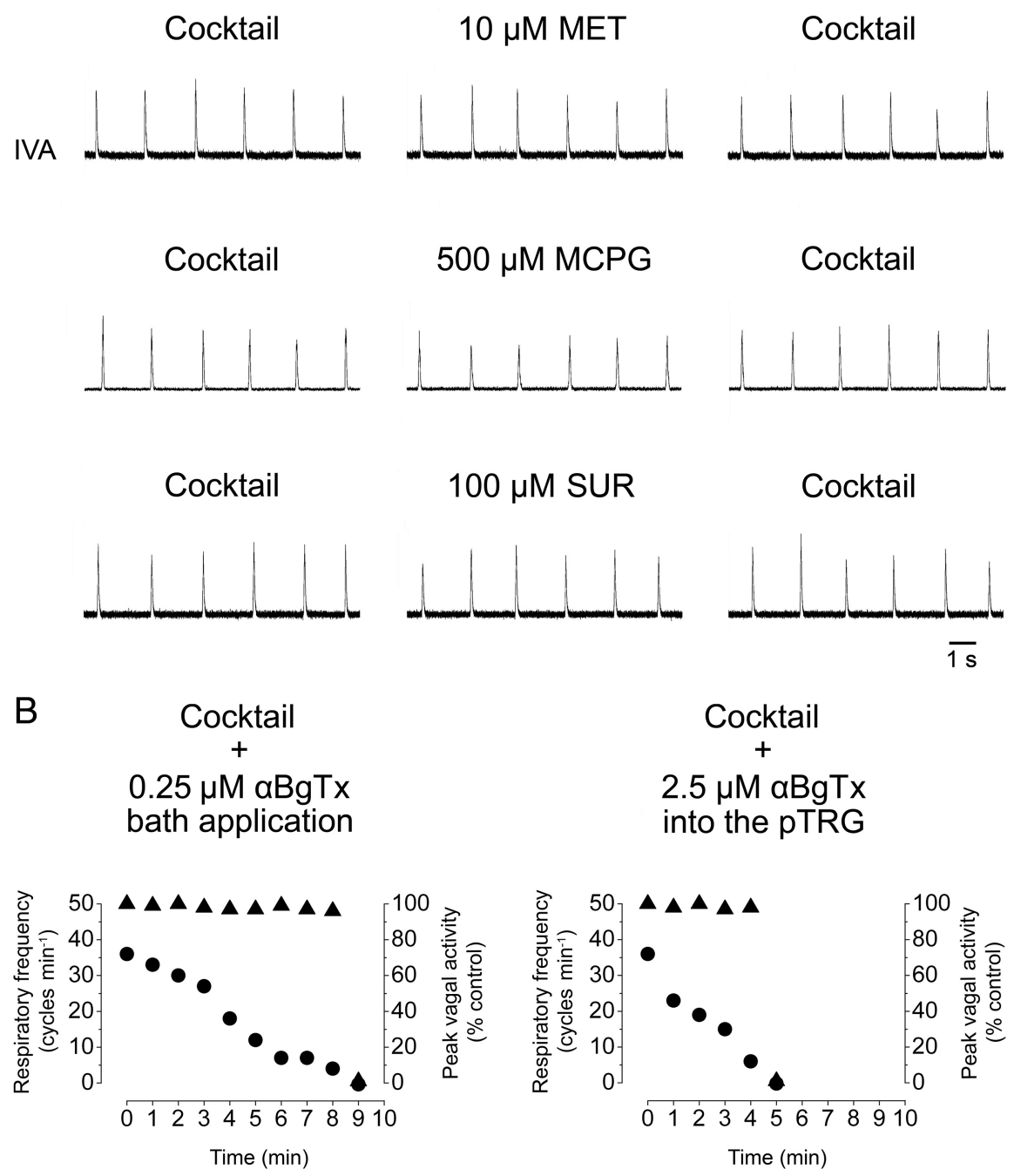

Figure 5. Role of nicotinic receptors in maintaining the respiratory rhythm during blockade of $\mathrm{iGlu}, \mathrm{GABA}_{\mathrm{A}}$, and glycine receptors (cocktail). The cocktail contains $20 \mu \mathrm{M}$ CNQX, $100 \mu \mathrm{M}$ D-AP5, $10 \mu \mathrm{m}$ bicuculline (Bic), and $10 \mu \mathrm{m}$ strychnine (Stryc). $A$, No change in integrated vagal nerve activity (IVA) $\sim 10$ min after bath application of $10 \mu \mathrm{m}$ methysergide (MET), $500 \mu \mathrm{M} \mathrm{MCPG}$, or $100 \mu \mathrm{m}$ suramin (SUR). B, Bath application of $0.25 \mu \mathrm{m} \alpha$-bungarotoxin ( $\alpha \mathrm{BgTx}$ ) and microinjections of $2.5 \mu \mathrm{M} \alpha \mathrm{BgTx}$ into the pTRG abolished respiratory rhythmic activity within $\sim 10$ and $5 \mathrm{~min}$, respectively. Examples of these effects are illustrated as time course of changes in peak amplitude $(\boldsymbol{\Delta})$ and frequency $(\boldsymbol{O})$ of vagal activity.

the main contribution to respiratory rhythm generation derives from disinhibition of cholinergic neurons because of the possibly incomplete but intense blockade of iGluRs. Finally, it seems unlikely that disinhibition could affect mainly glutamatergic neurons and cause increases in the release of Glu in the presence of an $\mathrm{ACh}$ release similar to that present under control perfusing conditions.

\section{Discussion}

We have shown that ACh plays an important excitatory role within the lamprey respiratory network under basal conditions and possibly under still undefined physiological conditions that imply increased respiratory drive to vagal motoneurons such as locomotion or enhanced sensory inputs (Gravel et al., 2007; Gariepy et al., 2010). This excitatory role is mediated by $\alpha 7$ nAChRs within the pTRG. Blockade of iGluRs at concentrations up to $100 \mu \mathrm{M}$ CNQX and $100 \mu \mathrm{M}$ D-AP5 is not complete but sufficient to arrest the respiratory CPG and, as a consequence, vagal motor output, despite that glutamatergic transmission is still, to some extent, effective at the level of vagal motoneurons. In addition, the respiratory motor output, although at reduced frequency, persists during blockade of iGluRs when also $\mathrm{GABA}_{\mathrm{A}}$ and glycine receptors are blocked. This respiratory activity is suppressed by blockade of pTRG $\alpha 7$ nAChRs, thus suggesting that under these conditions ACh per se is capable of maintaining the respiratory rhythm. All the respiratory responses to ACh-related drugs are mediated at the level of the PTRG, thus confirming its importance in respiratory rhythm generation.

The finding that ACh has an excitatory effect on respiration is consistent with previous results obtained in mammals (Gesell et al., 1943; Murakoshi et al., 1985; Monteau et al., 1990; Burton et al., 1994, 1995; Bianchi et al., 1995; Shao and Feldman, 2000, 2001, 2002, 2005, 2009; Hatori et al., 2006; Shao et al., 2008). Noticeably, the present findings show that ACh affects respiratory activity through a specific action on the pTRG. Cholinergic inputs to the pTRG may arise from several regions of the lamprey brain where cholinergic neurons have been mapped (Pombal et al., 2001; Le Ray et al., 2003). In the brainstem, choline acetyltransferaseimmunoreactive neurons include various groups of cranial motoneurons and two distinct groups of neurons in the tegmentum at the mesopontine border, in an area encompassing the isthmus and the caudal mesencephalon. In particular, immunoreactive cells have been encountered close to the isthmic Müller cells located in the anterior rhombencephalic reticular nucleus. The latter appears to be in close proximity with or even within the pTRG (Fig. 2; for comparison, see Pombal et al., 2001; Le Ray et al., 2003). Cholinergic neurons have also been encountered in the mesencephalic locomotor region. Their inputs induce depolarization of reticulospinal cells involved in the initiation and control of locomotion through an action on $\alpha 7$ nAChRs (Pombal et al., 2001; Le Ray et al., 2003). Reasonably, the hypothesis can be advanced that the mesencephalic locomotor region conveys similar cholinergic inputs to the PTRG in response to locomotion-inducing stimuli that imply a parallel stimulation of respiratory activity.

The respiratory effects induced by $\alpha 7 \mathrm{nAChR}$ blockade indicate that during fictive breathing in vitro $\alpha 7 \mathrm{nAChRs}$ are activated by endogenously released $\mathrm{ACh}$ and have a role in the modulation of baseline respiratory activity. It seems unlikely that endogenous $\mathrm{ACh}$ arises from cranial motoneurons or from the mesencephalic locomotor region. The latter is brought into action under particular circumstances, but not under resting conditions. Most probably, ACh is released from neurons located in close proximity with or even within the pTRG. Varied results have been reported 
on the modulatory role of endogenously released ACh in in vitro rodent preparations (Murakoshi et al., 1985; Monteau et al., 1990; Burton et al., 1994, 1995; Shao and Feldman, 2000, 2001, 2002, 2005; Hatori et al., 2006). In contrast with these previous results, we show that ACh affects respiration in the lamprey by acting solely on nAChRs. The lack of respiratory effects in response to bath application of pilocarpine and atropine indicates that $\mathrm{mAChRs}$ do not contribute to respiratory regulation, although are involved in the control of lamprey locomotion (Le Ray et al., 2004; Smetana et al., 2010). In contrast with previous findings concerning the preBötC of neonatal rats (Shao and Feldman, 2002; Shao et al., 2008), we found that only $\alpha 7 \mathrm{nAChRs}$ are expressed within the pTRG. These discrepancies may depend on differences in the animal species used. Recent neuroanatomical studies have shown that $\alpha 7 \mathrm{nAChRs}$ are more common in the preBötC region than electrophysiological studies would suggest, but their role remains to be elucidated (Dehkordi et al., 2004). In agreement with the general notion that nAChRs can presynaptically modulate both glutamatergic and GABAergic neurotransmission (Fregosi and Pilarski, 2008; Albuquerque et al., 2009), evidence has been provided that nicotine acts on $\alpha 4 \beta 2$ nAChRs of preBötC inspiratory neurons by modulating glutamatergic neurotransmission (Shao and Feldman, 2001). Although information is lacking on the cellular localization of nAChRs in the lamprey respiratory network, the finding that nicotine-induced activation still produces respiratory effects during partial, but relatively intense, blockade of iGluRs does not support the hypothesis of a presynaptic localization of $\alpha 7$ nAChRs on glutamatergic neurons. Present results show that activation or blockade of $\alpha 7 \mathrm{nAChRs}$ causes changes in respiratory frequency without concomitant variations in the amplitude of vagal bursts, thus suggesting that separate mechanisms are involved in the timing and intensity control of respiratory activity. In agreement with the view that changes in respiratory frequency are due to an action on the central mechanisms generating the respiratory rhythm (Feldman and Del Negro, 2006; Mutolo et al., 2010), respiratory responses induced by bath application of $\mathrm{nAChR}$ agonists or antagonists were mimicked by microinjections of the same drugs into the pTRG.

The finding that the respiratory rhythm persists following cocktail application is surprising (Tryba et al., 2003; Peña et al., 2004; Del Negro et al., 2005). Present results confirm that application of iGluR antagonists at appropriate concentrations (Bongianni et al., 1999) abolishes the respiratory motor output and provide evidence that respiratory activity is restored by subsequent blockade of $\mathrm{GABA}_{\mathrm{A}}$ and glycine receptors. However, they also show that glutamatergic transmission was not completely blocked at concentrations sufficient to arrest the respiratory rhythm of pTRG neurons and, therefore, their excitatory drive to vagal motoneurons. Respiratory neurons of the pTRG, unlike vagal motoneurons, proved to be responsive to the activation or blockade of $\alpha 7 \mathrm{nAChRs}$, thus indicating that nAChR-expressing neurons of the pTRG contribute to respiratory rhythm generation after cocktail application. It should also be mentioned that $\alpha 7 \mathrm{nAChR}$ blockade and bath application of the cocktail solution increased the duration of vagal bursts. The reasons of these outcomes are obscure. The absence of the inhibitory control per se does not consistently affect burst duration (Bongianni et al., 2006). Thus, it can be suggested that reductions in the excitatory input to pTRG neurons could decrease the excitatory coupling between pTRG neurons resulting in an increase in the duration of spontaneous vagal bursts (Shao and Feldman, 2002). Reductions in the excitatory coupling between vagal motoneurons (if any) as well as in the efficacy of segmental excitatory and inhibitory Renshaw-like mechanisms controlling motoneuron discharge may have played a role in the observed effects (Rovainen, 1974; Parker, 2003; Quinlan and Buchanan, 2008).

The removal of the modulatory control exerted by the inhibitory mechanisms (Bongianni et al., 2006) causes obvious disinhibition phenomena that could involve an increased release of $\mathrm{ACh}$ and/or Glu. Disinhibition of cholinergic mechanisms is suggested by their essential role during cocktail perfusion (Figs. $5 B$, $6 C)$. However, a portion of iGluRs is still active during cocktail application; thus, we cannot exclude that an increase in Glu release may have contributed to respiratory rhythm resumption. The possibility therefore exists that both cholinergic and glutamatergic mechanisms are necessary for the resumption of respi- 
ratory rhythmicity. An attractive hypothesis is that neurons belonging to the PTRG display colocalization of ACh and Glu (Grillner and Jessell, 2009). Models of possible synaptic interactions within the PTRG were proposed in Results. It seems unlikely that tonic cholinergic inputs may act by raising the excitability of rhythmogenic glutamatergic neurons, since the resumption of the respiratory rhythm can occur in the presence of a relatively intense blockade of iGluRs.

In conclusion, we propose that these cholinergic neurons are possible components of the respiratory rhythmogenic network. They may have a modulatory function under basal conditions when glutamatergic mechanisms are essential (Bongianni et al., 1999) and a subsidiary but crucial rhythmogenic role when glutamatergic transmission is impaired. The presence of different rhythm-generating mechanisms could represent a safety factor and possibly allow the respiratory network to adjust to changes in the behavioral, environmental, and metabolic states (Peña et al., 2004). The results encourage further studies on the respiratory role of cholinergic mechanisms in different animal species.

\section{References}

Aberger K, Chitravanshi VC, Sapru HN (2001) Cardiovascular responses to microinjections of nicotine into the caudal ventrolateral medulla of the rat. Brain Res 892:138-146.

Albuquerque EX, Pereira EF, Alkondon M, Rogers SW (2009) Mammalian nicotinic acetylcholine receptors: from structure to function. Physiol Rev 89:73-120.

Auclair F, Lund JP, Dubuc R (2004) Immunohistochemical distribution of tachykinins in the CNS of the lamprey Petromyzon marinus. J Comp Neurol 479:328-346.

Bianchi AL, Denavit-Saubie M, Champagnat J (1995) Central control of breathing in mammals: neuronal circuitry, membrane properties, and neurotransmitters. Physiol Rev 75:1-45.

Bongianni F, Deliagina TG, Grillner S (1999) Role of glutamate receptor subtypes in the lamprey respiratory network. Brain Res 826:298-302.

Bongianni F, Mutolo D, Carfi M, Pantaleo T (2002) Group I and II metabotropic glutamate receptors modulate respiratory activity in the lamprey. Eur J Neurosci 16:454-460.

Bongianni F, Mutolo D, Nardone F, Pantaleo T (2006) GABAergic and glycinergic inhibitory mechanisms in the lamprey respiratory control. Brain Res 1090:134-145.

Brocard F, Bardy C, Dubuc R (2005) Modulatory effect of substance P to the brain stem locomotor command in lampreys. J Neurophysiol 93:2127-2141.

Burton MD, Nouri K, Baichoo S, Samuels-Toyloy N, Kazemi H (1994) Ventilatory output and acetylcholine: perturbations in release and muscarinic receptor activation. J Appl Physiol 77:2275-2284.

Burton MD, Nouri M, Kazemi H (1995) Acetylcholine and central respiratory control: perturbations of acetylcholine synthesis in the isolated brainstem of the neonatal rat. Brain Res 670:39-47.

Dehkordi O, Haxhiu MA, Millis RM, Dennis GC, Kc P, Jafri A, Khajavi M, Trouth CO, Zaidi SI (2004) Expression of alpha-7 nAChRs on spinal cord-brainstem neurons controlling inspiratory drive to the diaphragm. Respir Physiol Neurobiol 141:21-34.

Del Negro CA, Morgado-Valle C, Hayes JA, Mackay DD, Pace RW, Crowder EA, Feldman JL (2005) Sodium and calcium current-mediated pacemaker neurons and respiratory rhythm generation. J Neurosci 25:446-453.

Doi A, Ramirez JM (2008) Neuromodulation and the orchestration of the respiratory rhythm. Respir Physiol Neurobiol 164:96-104.

Feldman JL, Del Negro CA (2006) Looking for inspiration: new perspectives on respiratory rhythm. Nat Rev Neurosci 7:232-242.

Fregosi RF, Pilarski JQ (2008) Prenatal nicotine exposure and development of nicotinic and fast amino acid-mediated neurotransmission in the control of breathing. Respir Physiol Neurobiol 164:80-86.

Gariepy JF, Missaghi K, Dubuc R (2010) The interactions between locomotion and respiration. Prog Brain Res 187:173-188.

Gesell R, Hansen ET, Worzniak JJ (1943) Humoral intermediation of nerve cell activation in the central nervous system. Am J Physiol 138:776-791

Gravel J, Brocard F, Gariepy JF, Lund JP, Dubuc R (2007) Modulation of respiratory activity by locomotion in lampreys. Neuroscience 144 $1120-1132$.

Grillner S (2006) Biological pattern generation: the cellular and computational logic of networks in motion. Neuron 52:751-766.

Grillner S, Jessell TM (2009) Measured motion: searching for simplicity in spinal locomotor networks. Curr Opin Neurobiol 19:572-586.

Guimond JC, Auclair F, Lund JP, Dubuc R (2003) Anatomical and physiological study of respiratory motor innervation in lampreys. Neuroscience 122:259-266

Hatori E, Sakuraba S, Kashiwagi M, Kuribayashi J, Tsujita M, Hosokawa Y, Takeda J, Kuwana S (2006) Association of nicotinic acetylcholine receptors with central respiratory control in isolated brainstem-spinal cord preparation of neonatal rats. Biol Res 39:321-330.

Hotton N (1976) Origin and radiation of poikilothermous vertebrates. In: Evolution of brain and behavior in vertebrates (Masterton RB, Campbel CBG, Bitterman ME, Hotton N, eds), pp 1-24. Hillsdale, NJ: Erlbaum.

Le Ray D, Brocard F, Bourcier-Lucas C, Auclair F, Lafaille P, Dubuc R (2003) Nicotinic activation of reticulospinal cells involved in the control of swimming in lampreys. Eur J Neurosci 17:137-148.

Le Ray D, Brocard F, Dubuc R (2004) Muscarinic modulation of the trigemino-reticular pathway in lampreys. J Neurophysiol 92:926938.

Lorier AR, Huxtable AG, Robinson DM, Lipski J, Housley GD, Funk GD (2007) P2Y1 receptor modulation of the pre-Bötzinger complex inspiratory rhythm generating network in vitro. J Neurosci 27:9931005.

Monteau R, Morin D, Hilaire G (1990) Acetylcholine and central chemosensitivity: in vitro study in the newborn rat. Respir Physiol $81: 241-253$.

Murakoshi T, Suzue T, Tamai S (1985) A pharmacological study on respiratory rhythm in the isolated brainstem-spinal cord preparation of the newborn rat. Br J Pharmacol 86:95-104.

Mutolo D, Bongianni F, Einum J, Dubuc R, Pantaleo T (2007) Opioidinduced depression in the lamprey respiratory network. Neuroscience 150:720-729.

Mutolo D, Bongianni F, Cinelli E, Pantaleo T (2010) Role of neurokinin receptors and ionic mechanisms within the respiratory network of the lamprey. Neuroscience 169:1136-1149.

Parker D (2003) Activity-dependent feedforward inhibition modulates synaptic transmission in a spinal locomotor network. J Neurosci 23:11085-11093.

Peña F, Parkis MA, Tryba AK, Ramirez JM (2004) Differential contribution of pacemaker properties to the generation of respiratory rhythms during normoxia and hypoxia. Neuron 43:105-117.

Pombal MA, Marin O, Gonzalez A (2001) Distribution of choline acetyltransferase-immunoreactive structures in the lamprey brain. J Comp Neurol 431:105-126.

Quinlan KA, Buchanan JT (2008) Cellular and synaptic actions of acetylcholine in the lamprey spinal cord. J Neurophysiol 100:1020-1031.

Rovainen CM (1974) Respiratory motoneurons in lampreys. J Comp Physiol 94:57-68.

Rovainen CM (1977) Neural control of ventilation in the lamprey. Fed Proc $36: 2386-2389$.

Rovainen CM (1979) Neurobiology of lampreys. Physiol Rev 59:1007-1077.

Rovainen CM (1983) Generation of respiratory activity by the lamprey brain exposed to picrotoxin and strychnine, and weak synaptic inhibition in motoneurons. Neuroscience 10:875-882.

Rovainen CM (1985) Respiratory bursts at the midline of the rostral medulla of the lamprey. J Comp Physiol A Neuroethol Sens Neural Behav Physiol 157:303-309.

Russell DF (1986) Respiratory pattern generation in adult lampreys (Lampetra fluviatilis): interneurons and burst resetting. J Comp Physiol A Neuroethol Sens Neural Behav Physiol 158:91-102.

Shao XM, Feldman JL (2000) Acetylcholine modulates respiratory pattern: effects mediated by M3-like receptors in preBötzinger complex inspiratory neurons. J Neurophysiol 83:1243-1252.

Shao XM, Feldman JL (2001) Mechanisms underlying regulation of respi- 
ratory pattern by nicotine in preBötzinger complex. J Neurophysiol 85:2461-2467.

Shao XM, Feldman JL (2002) Pharmacology of nicotinic receptors in preBötzinger complex that mediate modulation of respiratory pattern. J Neurophysiol 88:1851-1858.

Shao XM, Feldman JL (2005) Cholinergic neurotransmission in the preBötzinger complex modulates excitability of inspiratory neurons and regulates respiratory rhythm. Neuroscience 130:1069-1081.

Shao XM, Feldman JL (2009) Central cholinergic regulation of respiration: nicotinic receptors. Acta Pharmacol Sin 30:761-770.

Shao XM, Tan W, Xiu J, Puskar N, Fonck C, Lester HA, Feldman JL (2008) Alpha4 ${ }^{*}$ nicotinic receptors in preBötzinger complex mediate cholinergic/ nicotinic modulation of respiratory rhythm. J Neurosci 28:519-528.

Smetana R, Juvin L, Dubuc R, Alford S (2010) A parallel cholinergic brainstem pathway for enhancing locomotor drive. Nat Neurosci 13:731-738.

Smith JC, Ellenberger HH, Ballanyi K, Richter DW, Feldman JL (1991) PreBötzinger complex: a brainstem region that may generate respiratory rhythm in mammals. Science 254:726-729.

St. John WM, Leiter JC (2008) Maintenance of gasping and restoration of eupnea after hypoxia is impaired following blockers of alpha1adrenergic receptors and serotonin 5-HT2 receptors. J Appl Physiol 104:665-673.

Thompson KJ (1985) Organization of inputs to motoneurons during fictive respiration in the isolated lamprey brain. J Comp Physiol A Neuroethol Sens Neural Behav Physiol 157:291-302.

Tryba AK, Pena F, Ramirez JM (2003) Stabilization of bursting in respiratory pacemaker neurons. J Neurosci 23:3538-3546. 